A N N A LES

UNIVERSITATIS MARIAE CURIE-SKŁODOWSKA

LUBLIN - POLONIA

VOL. LXII, 2008

SECTIO A

$105-111$

ANDRZEJ MIERNOWSKI

\title{
Parallelograms inscribed in a curve having a circle as $\frac{\pi}{2}$-isoptic
}

\begin{abstract}
Jean-Marc Richard observed in [7] that maximal perimeter of a parallelogram inscribed in a given ellipse can be realized by a parallelogram with one vertex at any prescribed point of ellipse. Alain Connes and Don Zagier gave in [4] probably the most elementary proof of this property of ellipse. Another proof can be found in [1]. In this note we prove that closed, convex curves having circles as $\frac{\pi}{2}$-isoptics have the similar property.
\end{abstract}

1. Introduction. Let $C$ be a closed and strictly convex curve. We fix an interior point of $C$ as an origin of a coordinate system. Denote $e^{i t}=$ $(\cos t, \sin t), i e^{i t}=(-\sin t, \cos t)$. The function $p: \mathbb{R} \rightarrow \mathbb{R}$

$$
p(t)=\sup _{z \in C}\left\langle z, e^{i t}\right\rangle
$$

is called the support function of $C$. For a strictly convex curve $p$ is differentiable. We assume that the function $p$ is of class $C^{2}$ and the curvature of $C$ is positive. We have the following equation of $C$ in terms of its support function

$$
z(t)=p(t) e^{i t}+\dot{p}(t) i e^{i t} .
$$

Then $\|z\|=\sqrt{p^{2}(t)+\dot{p}^{2}(t)}$ and $R(t)=p(t)+\ddot{p}(t)$ is a radius of curvature of $C$ at $t$.

2000 Mathematics Subject Classification. 53C12.

Key words and phrases. Convex curve, support function, curvature. 
The $\alpha$-isoptic of $C$ consists of those points in the plane from which the curve is seen under the fixed angle $\alpha$ (for the geometric properties of isoptics see [2], [3], [5], [6], [8]). Suppose that $\frac{\pi}{2}$-isoptic of $C$ is a circle of radius $r$ with the center in the origin of a coordinate system. Then

$$
p^{2}(t)+p^{2}\left(t+\frac{\pi}{2}\right)=r^{2},
$$

and

$$
p^{2}(t+\pi)+p^{2}\left(t+\frac{\pi}{2}\right)=r^{2},
$$

so $p(t)=p(t+\pi)$ and the center of the circle is a center of symmetry of $C$. The curve (1.1) has a circle with the center in the origin of a coordinate system as an $\frac{\pi}{2}$-isoptic if and only if (1.2) holds good.

Example 1.1. Let $C$ be an ellipse $\frac{x^{2}}{a^{2}}+\frac{y^{2}}{b^{2}}=1$. Then

$$
\begin{gathered}
p(t)=\sqrt{a^{2} \cos ^{2} t+b^{2} \sin ^{2} t}, \\
z(t)=(x(t), y(t))=\sqrt{a^{2} \cos ^{2} t+b^{2} \sin ^{2} t} e^{i t}+\frac{\sin t \cos t\left(b^{2}-a^{2}\right)}{\sqrt{a^{2} \cos ^{2} t+b^{2} \sin ^{2} t}} i e^{i t}
\end{gathered}
$$

is its equation in terms of a support function and $p^{2}(t)+p^{2}\left(t+\frac{\pi}{2}\right)=a^{2}+b^{2}$.

2. Extremal property of the perimeter of inscribed parallelograms. Assume that a curve $C$ given by (1.1) has a circle with a center in an origin of a coordinate system as an $\frac{\pi}{2}$-isoptic. Then we have (1.2) and

$$
p(t) \dot{p}(t)+p\left(t+\frac{\pi}{2}\right) \dot{p}\left(t+\frac{\pi}{2}\right)=0 .
$$

Fix $t$ and consider inscribed parallelogram with $z(t)$ as one of the vertices. There exists $\alpha$ such that $z(t+\alpha),-z(t),-z(t+\alpha)$ are its remaining vertices and

$$
d_{t}(\alpha)=|z(t+\alpha)-z(t)|+|z(t+\alpha)+z(t)|
$$

is a half of a perimeter of parallelogram.

Theorem 2.1. Let $C$ be a strictly convex curve having a circle with a center in an origin of a coordinate system as an $\frac{\pi}{2}$-isoptic and let $d_{t}(\alpha)$ be the function given by (2.2). Then

(i) $d_{t}^{\prime}\left(\frac{\pi}{2}\right)=0$, where prime denotes the derivative with respect to $\alpha$,

(ii) $d\left(\frac{\pi}{2}\right)=d_{t}\left(\frac{\pi}{2}\right)$ does not depend on $t$.

Proof. We have

$$
\begin{aligned}
e^{i(t+\alpha)}= & \cos \alpha e^{i t}+\sin \alpha i e^{i t} \\
i e^{i(t+\alpha)}= & -\sin \alpha e^{i t}+\cos \alpha i e^{i t}, \\
z(t+\alpha)= & (p(t+\alpha) \cos \alpha-\dot{p}(t+\alpha) \sin \alpha) e^{i t} \\
& +(p(t+\alpha) \sin \alpha+\dot{p}(t+\alpha) \cos \alpha) i e^{i t} .
\end{aligned}
$$


Let

$$
\begin{aligned}
& A=p(t+\alpha) \cos \alpha-\dot{p}(t+\alpha) \sin \alpha-p(t), \\
& B=p(t+\alpha) \sin \alpha+\dot{p}(t+\alpha) \cos \alpha-\dot{p}(t), \\
& C=p(t+\alpha) \cos \alpha-\dot{p}(t+\alpha) \sin \alpha+p(t), \\
& D=p(t+\alpha) \sin \alpha+\dot{p}(t+\alpha) \cos \alpha+\dot{p}(t) .
\end{aligned}
$$

Then

$$
d_{t}(\alpha)=\sqrt{A^{2}+B^{2}}+\sqrt{C^{2}+D^{2}}
$$

and

$$
\begin{aligned}
& d_{t}^{\prime}(\alpha)=(p(t+\alpha)+\ddot{p}(t+\alpha)) \\
& \times\left(\frac{\dot{p}(t+\alpha)+p(t) \sin \alpha-\dot{p}(t) \cos \alpha}{\sqrt{A^{2}+B^{2}}}+\frac{\dot{p}(t+\alpha)-p(t) \sin \alpha+\dot{p}(t) \cos \alpha}{\sqrt{C^{2}+D^{2}}}\right) .
\end{aligned}
$$

Putting $\alpha=\frac{\pi}{2}$, we get

$$
\begin{gathered}
d_{t}^{\prime}\left(\frac{\pi}{2}\right)=R\left(t+\frac{\pi}{2}\right)\left(\frac{\dot{p}\left(t+\frac{\pi}{2}\right)+p(t)}{\sqrt{\left(p(t)+\dot{p}\left(t+\frac{\pi}{2}\right)\right)^{2}+\left(p\left(t+\frac{\pi}{2}\right)-\dot{p}(t)\right)^{2}}}\right. \\
\left.+\frac{\dot{p}\left(t+\frac{\pi}{2}\right)-p(t)}{\sqrt{\left(p(t)-\dot{p}\left(t+\frac{\pi}{2}\right)\right)^{2}+\left(p\left(t+\frac{\pi}{2}\right)+\dot{p}(t)\right)^{2}}}\right) .
\end{gathered}
$$

From (2.1) we have

$$
\dot{p}\left(t+\frac{\pi}{2}\right)=-\frac{p(t) \dot{p}(t)}{p\left(t+\frac{\pi}{2}\right)},
$$

and since

$$
\begin{aligned}
\left(p\left(t+\frac{\pi}{2}\right)-\dot{p}(t)\right) & \left(p\left(t+\frac{\pi}{2}\right)+\dot{p}(t)\right) \\
= & p^{2}\left(t+\frac{\pi}{2}\right)-\dot{p}^{2}(t)=r^{2}-\left(p^{2}(t)+\dot{p}^{2}(t)\right) \\
= & r^{2}-\|z(t)\|^{2}>0
\end{aligned}
$$

we obtain

$$
\operatorname{sgn}\left(p\left(t+\frac{\pi}{2}\right)-\dot{p}(t)\right)=\operatorname{sgn}\left(p\left(t+\frac{\pi}{2}\right)+\dot{p}(t)\right) .
$$

Hence

$$
\begin{aligned}
& \frac{\dot{p}\left(t+\frac{\pi}{2}\right)+p(t)}{\sqrt{\left(p(t)+\dot{p}\left(t+\frac{\pi}{2}\right)\right)^{2}+\left(p\left(t+\frac{\pi}{2}\right)-\dot{p}(t)\right)^{2}}} \\
& +\frac{\dot{p}\left(t+\frac{\pi}{2}\right)-p(t)}{\sqrt{\left(p(t)-\dot{p}\left(t+\frac{\pi}{2}\right)\right)^{2}+\left(p\left(t+\frac{\pi}{2}\right)+\dot{p}(t)\right)^{2}}}
\end{aligned}
$$




$$
\begin{gathered}
=\frac{-\frac{p(t) \dot{p}(t)}{p\left(t+\frac{\pi}{2}\right)}+p(t)}{\sqrt{\left(p(t)-\frac{p(t) \dot{p}(t)}{p\left(t+\frac{\pi}{2}\right)}\right)^{2}+\left(p\left(t+\frac{\pi}{2}\right)-\dot{p}(t)\right)^{2}}} \\
+\frac{-\frac{p(t) \dot{p}(t)}{p\left(t+\frac{\pi}{2}\right)}-p(t)}{\sqrt{\left(p(t)+\frac{p(t) \dot{p}(t)}{p\left(t+\frac{\pi}{2}\right)}\right)^{2}+\left(p\left(t+\frac{\pi}{2}\right)+\dot{p}(t)\right)^{2}}} \\
=\frac{p(t)}{p\left(t+\frac{\pi}{2}\right)}\left(\frac{\left(p\left(t+\frac{\pi}{2}\right)-\dot{p}(t)\right) p\left(t+\frac{\pi}{2}\right)}{\left|p\left(t+\frac{\pi}{2}\right)-\dot{p}(t)\right| \sqrt{p^{2}(t)+p^{2}\left(t+\frac{\pi}{2}\right)}}\right. \\
\left.-\frac{\left(p\left(t+\frac{\pi}{2}\right)+\dot{p}(t)\right) p\left(t+\frac{\pi}{2}\right)}{\left|p\left(t+\frac{\pi}{2}\right)+\dot{p}(t)\right| \sqrt{p^{2}(t)+p^{2}\left(t+\frac{\pi}{2}\right)}}\right)=0,
\end{gathered}
$$

which proves the first part of Theorem 2.1.

Let

$$
\begin{aligned}
h(t)=d_{t}\left(\frac{\pi}{2}\right)= & \sqrt{\left(p(t)+\dot{p}\left(t+\frac{\pi}{2}\right)\right)^{2}+\left(\dot{p}(t)-p\left(t+\frac{\pi}{2}\right)\right)^{2}} \\
& +\sqrt{\left(p(t)-\dot{p}\left(t+\frac{\pi}{2}\right)\right)^{2}+\left(\dot{p}(t)+p\left(t+\frac{\pi}{2}\right)\right)^{2}} .
\end{aligned}
$$

Then

$$
\begin{aligned}
& \dot{h}(t)= \frac{R(t)\left(\dot{p}(t)-p\left(t+\frac{\pi}{2}\right)\right)+R\left(t+\frac{\pi}{2}\right)\left(p(t)+\dot{p}\left(t+\frac{\pi}{2}\right)\right)}{\sqrt{\left(p(t)+\dot{p}\left(t+\frac{\pi}{2}\right)\right)^{2}+\left(\dot{p}(t)-p\left(t+\frac{\pi}{2}\right)\right)^{2}}} \\
&+ R\left(t+\frac{\pi}{2}\right)\left(\dot{p}\left(t+\frac{\pi}{2}\right)-p(t)\right)+R(t)\left(\dot{p}(t)+p\left(t+\frac{\pi}{2}\right)\right) \\
& \sqrt{\left(p(t)-\dot{p}\left(t+\frac{\pi}{2}\right)\right)^{2}+\left(\dot{p}(t)+p\left(t+\frac{\pi}{2}\right)\right)^{2}} \\
&=R\left(t+\frac{\pi}{2}\right)\left(\frac{p(t)+\dot{p}\left(t+\frac{\pi}{2}\right)}{\sqrt{\left(p(t)+\dot{p}\left(t+\frac{\pi}{2}\right)\right)^{2}+\left(\dot{p}(t)-p\left(t+\frac{\pi}{2}\right)\right)^{2}}}\right. \\
&+ \\
&\left.+\frac{p(t)-\dot{p}\left(t+\frac{\pi}{2}\right)}{\sqrt{\left(p(t)-\dot{p}\left(t+\frac{\pi}{2}\right)\right)^{2}+\left(\dot{p}(t)+p\left(t+\frac{\pi}{2}\right)\right)^{2}}}\right) \\
&\left.+\frac{\dot{p}(t)-p\left(t+\frac{\pi}{2}\right)}{\sqrt{\left(p(t)-\dot{p}\left(t+\frac{\pi}{2}\right)\right)^{2}+\left(\dot{p}(t)+p\left(t+\frac{\pi}{2}\right)\right)^{2}}}\right) \\
&
\end{aligned}
$$


Since the first summand is equal to zero for each $t$ and the second summand is equal to the first at $t+\frac{\pi}{2}$, they are equal to zero.

3. The converse theorem. In this section we shall prove the converse of Theorem 2.1. For this purpose we define the function $d(t)=d_{t}\left(\frac{\pi}{2}\right)$.

Theorem 3.1. Let $C$ be a closed and strictly convex curve of class $C^{2}$ with positive curvature having a center of symmetry. Suppose that an origin of a coordinate system is in the center of $C$ and $d_{t}^{\prime}\left(\frac{\pi}{2}\right)=0$. Then $\dot{d}(t)=0$ and $\frac{\pi}{2}$-isoptic of $C$ is a circle.

Proof. The equality $d_{t}^{\prime}\left(\frac{\pi}{2}\right)=0$ is equivalent to

$$
\begin{aligned}
& \frac{\dot{p}\left(t+\frac{\pi}{2}\right)+p(t)}{\sqrt{\left(p(t)+\dot{p}\left(t+\frac{\pi}{2}\right)\right)^{2}+\left(p\left(t+\frac{\pi}{2}\right)-\dot{p}(t)\right)^{2}}} \\
& =\frac{p(t)-\dot{p}\left(t+\frac{\pi}{2}\right)}{\sqrt{\left(p(t)-\dot{p}\left(t+\frac{\pi}{2}\right)\right)^{2}+\left(p\left(t+\frac{\pi}{2}\right)+\dot{p}(t)\right)^{2}}} .
\end{aligned}
$$

The equality (3.1) for $t+\frac{\pi}{2}$ gives

$$
\begin{aligned}
& \frac{\dot{p}(t)+p\left(t+\frac{\pi}{2}\right)}{\sqrt{\left(p\left(t+\frac{\pi}{2}\right)+\dot{p}(t)\right)^{2}+\left(p(t)-\dot{p}\left(t+\frac{\pi}{2}\right)\right)^{2}}} \\
& =\frac{p\left(t+\frac{\pi}{2}\right)-\dot{p}(t)}{\sqrt{\left(p\left(t+\frac{\pi}{2}\right)-\dot{p}(t)\right)^{2}+\left(p(t)+\dot{p}\left(t+\frac{\pi}{2}\right)\right)^{2}}} .
\end{aligned}
$$

From (3.1) and (3.2) we get

or equivalently

$$
\frac{\dot{p}\left(t+\frac{\pi}{2}\right)+p(t)}{p\left(t+\frac{\pi}{2}\right)-\dot{p}(t)}=\frac{p(t)-\dot{p}\left(t+\frac{\pi}{2}\right)}{\dot{p}(t)+p\left(t+\frac{\pi}{2}\right)}
$$

$$
p(t) \dot{p}(t)+p\left(t+\frac{\pi}{2}\right)+p\left(t+\frac{\pi}{2}\right) \dot{p}\left(t+\frac{\pi}{2}\right)=0,
$$

which gives

$$
p^{2}(t)+p^{2}\left(t+\frac{\pi}{2}\right)=\text { const. }
$$

Example 3.1 $([5])$. Let $p(t)=\cos \left(\frac{\pi}{4}+k \sin (2 t)\right)$. For $k$ sufficiently small $p(t)$ is a support function of a closed and strictly convex curve having a circle as $\frac{\pi}{2}$-isoptic and different from an ellipse.

Example 3.2. Let $p(t)=\sqrt{a \sin ^{2} 3 t+b \cos ^{2} 9 t+c}$, for positive $a, b, c$. For $c$ sufficiently big $p(t)+\ddot{p}(t)>0$ for each $t$ and $p^{2}(t)+p^{2}\left(t+\frac{\pi}{2}\right)=a+b+2 c$ so $p(t)$ is a support function of a closed and strictly convex curve having a circle as $\frac{\pi}{2}$-isoptic. This curve cannot be an ellipse because an origin of a coordinate system is its center of symmetry and $p(t)$ is a periodic function 
with a period $\frac{\pi}{3}$. Hence its curvature function is also periodic with the same period and this curve has more then four vertices. More generally we can take $p(t)=\sqrt{a \sin ^{2} m t+b \cos ^{2} m n t+c}$, where $m$ and $m$ are odd integers and $a, b, c$ are positive.

Remark 3.1. Let $C$ be an ellipse. We fix a diameter $P P^{\prime}$ and consider an ellipse $C^{\prime}$ with focuses at $P$ and $P^{\prime}$ which is tangent to $C$. Then points $Q$ and $Q^{\prime}$ of tangency give a diameter such that a perimeter of parallelogram $P Q P^{\prime} Q^{\prime}$ is maximal. The common tangent of $C$ and $C^{\prime}$ at $Q$ (resp. $Q^{\prime}$ ) makes equal angels with the sides $P Q$ and $P^{\prime} Q$ (resp. $P Q^{\prime}$ and $P^{\prime} Q^{\prime}$ ). This means that for parallelogram of maximal perimeter a tangent at any vertex makes equal angles with adjoining sides. This is a part of a more general fact. Let $C$ be any closed and convex curve given in an arbitrary parametrization $z=z(t)$ of class $C^{1}$. Fix the points $z\left(t_{1}\right)$ and $z\left(t_{2}\right)$. Let $z\left(t_{0}\right)$ be such a point that the perimeter of the triangle $z\left(t_{1}\right) z\left(t_{2}\right) z\left(t_{0}\right)$ is maximal. Then the tangent at $t_{0}$ makes equal angels with the sides $z\left(t_{0}\right) z\left(t_{1}\right)$ and $z\left(t_{0}\right) z\left(t_{2}\right)$. Indeed,

$$
\begin{aligned}
\frac{d}{d t}(\mid z(t) & \left.-z\left(t_{1}\right)|+| z(t)-z\left(t_{2}\right) \mid\right) \\
= & \frac{\left\langle z(t)-z\left(t_{1}\right), \dot{z}(t)\right\rangle}{\left|z(t)-z\left(t_{1}\right)\right|}+\frac{\left\langle z(t)-z\left(t_{2}\right), \dot{z}(t)\right\rangle}{\left|z(t)-z\left(t_{2}\right)\right|} \\
= & \frac{\left|z(t)-z\left(t_{1}\right)\right||\dot{z}(t)| \cos \measuredangle\left(z(t)-z\left(t_{1}\right), \dot{z}(t)\right)}{\left|z(t)-z\left(t_{1}\right)\right|} \\
& +\frac{\left|z(t)-z\left(t_{2}\right)\right||\dot{z}(t)| \cos \measuredangle\left(z(t)-z\left(t_{2}\right), \dot{z}(t)\right)}{\left|z(t)-z\left(t_{2}\right)\right|} \\
= & |\dot{z}(t)|\left(\cos \measuredangle\left(z(t)-z\left(t_{1}\right), \dot{z}(t)\right)+\cos \measuredangle\left(z(t)-z\left(t_{2}\right), \dot{z}(t)\right)\right) .
\end{aligned}
$$

For $t=t_{0}$ we obtain

$$
\left.\cos \measuredangle\left(z\left(t_{0}\right)-z\left(t_{1}\right), \dot{z}\left(t_{0}\right)\right)+\cos \measuredangle\left(z\left(t_{0}\right)-z\left(t_{2}\right), \dot{z}\left(t_{0}\right)\right)\right)=0 .
$$

\section{REFERENCES}

[1] Berger, M., Geometrie, Vol. 2, Nathan, Paris, 1990.

[2] Cieślak, W., Miernowski, A. and Mozgawa, W., Isoptics of a strictly convex curve, Global Differential Geometry and Global Analysis, 1990 (Berlin), Lecture Notes in Math., 1481, Springer, Berlin, 1991, 28-35.

[3] Cieślak, W., Miernowski, A. and Mozgawa, W., Isoptics of a strictly convex curve II, Rend. Sem. Mat. Univ. Padova 96 (1996), 37-49.

[4] Connes, A., Zagier, D., A property of parallelograms inscribed in ellipses, Amer. Math. Monthly 114 (2007), 909-914.

[5] Green, J. W., Sets subtending a constant angle on a circle, Duke Math. J. 17 (1950), 263-267.

[6] Matsuura, S., On nonconvex curves of constant angle, Functional analysis and related topics, 1991 (Kyoto), Lecture Notes in Math., 1540, Springer, Berlin, 1993, 251-268.

[7] Richard, J-M., Safe domain and elementary geometry, Eur. J. Phys. 25 (2004), 835844. 
[8] Wunderlich, W., Kurven mit isoptischem Kreis, Aequationes Math. 6 (1971), 71-78.

\author{
Andrzej Miernowski \\ Institute of Mathematics \\ M. Curie-Skłodowska University \\ pl. Marii Curie-Skłodowskiej 1 \\ 20-031 Lublin, Poland \\ e-mail: mierand@hektor.umcs.lublin.pl
}

Received June 26, 2008 